\title{
Traumatic subarachnoid-pleural fistula
}

\author{
B. A. ROCHA CAMpos, L. B. Silva, N. BAllalai, AND M. M. NEGRÃO
}

From the Institute of Neurology and Neurosurgery, Londrina, Paraná, Brazil

SYNOPSIS The case of a child suffering from traumatic bilateral subarachnoid-pleural fistula is reported and the possible mechanisms responsible are discussed.

The occurrence of a fistula between the subarachnoid space and intrathoracic structures is uncommon. All cases so far reported in the literature (Wilson and Jamer, 1962; Bramwit and Schmelka, 1967; Overton et al., 1968; Zilkha et al., 1970; Cantu, 1971) have been due to trauma and the present case reported is the second in a child. In all cases the presence of such a fistula has been demonstrated at myelography by the recognition of contrast material in the thoracic cavity.

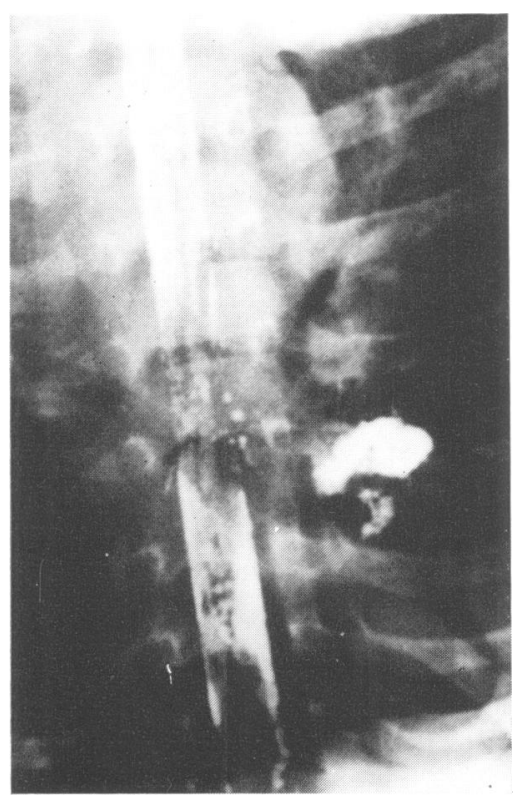

FIG. 1. Myelogram: frontal view. Shows Pantopaque outside the subarachnoid space at the level of T4-T5, on the right side, where the dural tear was larger.

\section{CASE HISTORY}

A.R.F., a 5 year old boy, was admitted to the Institute two hours after a car accident. On admission the systolic blood pressure was $50 \mathrm{mmHg}$ and the pulse rate 140 per minute. The child was alert but showed intense pallor with sweating and dyspnoea and he was complaining of pain in the chest and had a distended abdomen. There was some bruising of the thorax. Neurological examination showed the signs of paraplegia with motor and sensory deficits below the 5th thoracic segmental level. Radiographs of the chest revealed a right sided pleural effusion

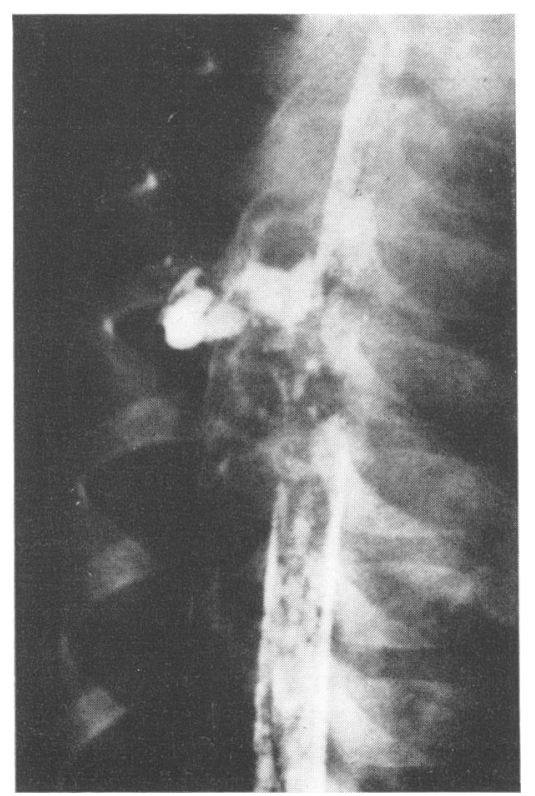

FIG. 2. Myelogram: lateral view. The Pantopaque column leaking to the pleural space. 
but there were no radiological abnormalities of the thoracic spine. During the next six hours the dyspnoea, chest pain, and abdominal distension became more marked and a Foley catheter was inserted because of retention of urine. The pleural effusion was tapped with the production of a slightly blood-stained fluid with a composition similar to cerebrospinal fluid (CSF).

Lumbar puncture was then performed: it showed a slightly blood-stained CSF with no manometric block. Three millilitres iophendylate (Pantopaque)

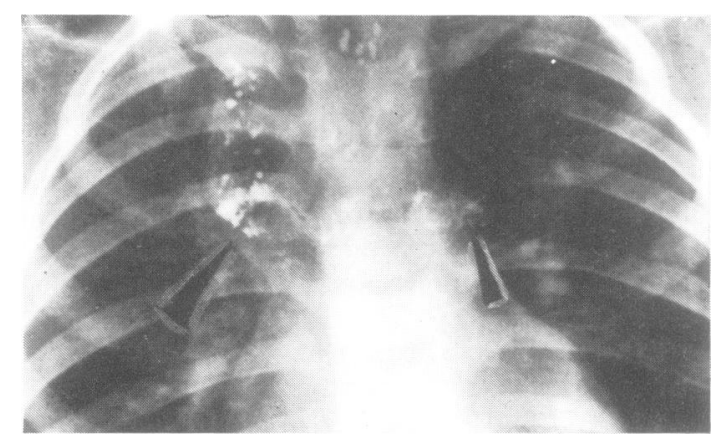

FIG. 3. Myelogram: frontal view. Contrast medium on the right and left side of the pleural cavities as shown by arrows.

were injected and myelographic screening was carried out. Streaming of Pantopaque into both pleural cavities was seen at the level of the $4 / 5$ thoracic vertebrae, more contrast medium passing to the right side than to the left (Figs 1, 2, and 3). On these findings the diagnosis of a thoracic subarachnoidpleural fistula was made.

OPERATION Laminectomy of T3, T4, and T5 vertebrae was carried out. The posterior surface of the dura mater was intact but the spinal cord was oedematous and showed petechial haemorrhages. There were tears of the dural root sleeves on both sides through which CSF could be seen dripping into the pleural cavity. The dural tears were repaired by suturing without grafts and the muscles were closed in multiple layers. Thoracotomy tubes were inserted on each side with water-seal suction. The thoracotomy tubes were removed five days after operation. Antibiotics were continued for a week and the Foley catheter was removed 10 days after the operation. There was immediate postoperative improvement in dyspnoea, chest pain, and abdominal distension but the neurological deficit has remained unchanged.

\section{DISCUSSION}

The mechanics of the injury by which such a fistula is produced are obscure and were considered by Zilkha et al. (1970). In those cases due to a penetrating wound presumably the missile itself is responsible for the perforation of the dura mater and the pleura. In the absence of penetration, as in the present case, a number of factors must be involved producing adjacent tears in both membranes. The thoracic spine and its emerging roots are relatively immobile structures and extreme dorsiflexion of the spine at the thoracic level could bring sufficient force on the root sleeve to cause tearing. In those cases reported as being due to blunt trauma (Overton et al., 1968; Zilkha et al., 1970; Cantu, 1971) there was sudden deceleration of the body on $\omega$ impact with a rigid structure. It seems likely tha $\$$ sudden and violent deceleration was responsibig for an extreme degree of flexion of the spine a well as for compression of the thoracic cage The accompanying thoracic compression causes perforation of the pleura on the anterior bon黑 prominences of the thoracic spine.

The indications for surgical treatment in these cases are related to the thoracic lesion rather than to the neurological one. In this case the child's general condition was greatly improved but, as in the other cases reported, the spinal cord lesion was unchanged. In all reported cases the diagnosis has been made at myelography, although it was strongly suspected in the present case on the discovery of CSF in the pleural cavities.

\section{REFERENCES}

Bramwit, D. N., and Schmelka, D. D. (1967). Traumatic subarachnoid-pleural fistula. Radiology, 89, 737-738.

Cantu, R. C. (1971). Value of myelography in thoracic spinal cord injuries. International Surgery, 56, 23-26.

Overton, M. C., III, Hood, R. M., and Farris, R. G. (1966). Traumatic subarachnoid-pleural fistula. Case report. Journal of Thoracic and Cardiovascular Surgery, 51, 729731

Wilson, C., and Jumer, M. (1962). Traumatic spinal-pleural N fistula. Journal of the American Medical Association, 179, 812-813.

Zilkha, A., Reiss, J., Shulman, K., and Schechter, M. M. (1970). Traumatic subarachnoid-mediastinal fistula. Case report. Journal of Neurosurgery, 32, 473-475. 